\title{
The Machine Translation of Literature: Implications for Translation Pedagogy
}

\author{
https://doi.org/10.3991/ijet.v15i11.13275 \\ Abdulfattah Omar ${ }^{(凶)}$ \\ Prince Sattam Bin Abdulaziz University, Al-Kharj, Saudi Arabia \\ a.abdelfattahepsau.edu.sa \\ Yasser A. Gomaa \\ Assiut University, Assiut, Egypt \\ University of Bahrain, Zallaq, Kingdom of Bahrain
}

\begin{abstract}
The recent years have witnessed an increasing importance of machine translation systems due to the prolific production on online texts in different disciplines and furthermore, the inability of traditional translation methods in addressing translation needs all over the world. It is even argued that training on translation tools should be integrated into translation pedagogies and ultimately, courses should be provided for students and professionals. In spite of the effectiveness of translation tools and systems in providing solutions in relation to different disciplines and text genres, the usability and reliability of such systems in terms of literary texts, however, is still highly controversial. Many critics and educators still underestimate the usefulness of the machine translation systems in literature, which could be partially attributed to the unique nature of the language of the literary texts. The issue has its pedagogical implications to translation instruction due to the needs to integrate emerging technologies in teaching and learning practices. For proper use of translation technologies in educational contexts, these need to be well evaluated. For this purpose, this study evaluates the usefulness of applying machine translation systems to literature with the purpose of identifying the challenges that may have negative impacts on the reliability of machine translation systems. In order to do this this, two translation systems are selected, namely, Google Translate and Q Translate. By way of illustration, the study is based on a corpus of two English short stories. The study is based on two prose fiction texts. The first is J. K. Rowling's novel Harry Potter and the Philosopher's Stone. The second is Edgar Allan Poe's short story The Black Cat. Automatic translations generated by the two machine translation systems were compared to human made Arabic translations with the purpose of identifying the problems within these translations. Results indicate that different lexical, structural, and pragmatic errors are encountered by users which negatively impact the reliability of these translations. Educators and translation instructors need to reflect on the challenges of machine translation systems in relation to literature. Software developers need also to address the problems faced by users and students in the translation from and into the Arabic language.
\end{abstract}


Keywords-Google Translate, literature, machine translation, QTranslate, translation pedagogy

\section{Introduction}

The recent years have witnessed the increasing importance of machine translation systems due to the prolific production on online texts in different disciplines and the inability of traditional translation methods in addressing translation needs all over the world [1-5]. It is even argued that translation tools and technologies should be integrated into translation pedagogies and additionally, courses should be provided for students and professionals [6-10]. In spite of the effectiveness of translation tools and systems in providing solutions in relation to different disciplines and text genres, the usability and reliability of such systems in terms of literary texts, nevertheless, is still controversial. Many critics, translators, and educators still underestimate the usefulness of the machine translation systems in literature. They typically point to the low quality or reliability of machine-translated literary texts [11-13]. This may be attributed to the unique nature of the language of the literary texts. The issue has its pedagogical implications to translation instruction due to the needs to integrate emerging technologies into teaching and consequently, learning practices $[14,15]$. For accurate use of translation technologies in educational contexts, these need to be well evaluated. For this purpose, this study evaluates the usefulness of applying machine translation systems to literature with the purpose of identifying the challenges that may have negative impacts on the reliability of machine translation systems. In order to do this this, two translation systems are selected. These are Google Translate and Q Translate. By way of illustration, the study is based on a corpus of two English short stories. The study is based on two prose fiction texts. The first is J. K. Rowling's novel Harry Potter and the Philosopher's Stone. The second is Edgar Allan Poe's short story The Black Cat. We are going to evaluate these translations by comparing them to our translation and other human made translation. Machine/ automatic translations generated by the three machine translation systems will be compared to human made translations with the purpose of identifying the problems within these translations.

\section{$2 \quad$ Literature Review}

Machine translation is an umbrella term that is used predominately for automatic translations of written and spoken data from one natural language to another using computers, software and applications. It can be defined as a translation from one natural language to another language through using the system of computers without the need or reliability of human intervention $[2,3,16]$. The history of machine translation is very old and it dates back to the $17^{\text {th }}$ century. The idea of machine translation appeared four hundred years ago [17]. It was not known as machine translation, instead, it was referred to as mechanical translation. Descartes was the first one to suggest that language can be represented throughout codes and words with various languages to give the same meaning when translated. He indicated that it is the procedure of modern machine 
technologies to form codified rules of the natural language throughout the designed machine. Moreover, Tong [18] asserts that the term automatic translation used instead of the term machine translation as a common term. Furthermore, Somers [19] indicated that, only after the Second World War, it was impossible to use stored program computers for the sake of language translation.

In spite of the increasing use of machine translation systems in different fields including the translation of academic articles, news, and even social media contents, there is a very limited work on applying machine translation to literature [20,21]. This may be attributed to the figurative devices, idioms humor and irony [22, 23]. Different studies, thus, have been concerned with evaluating machine translation systems in relation to literary texts including novels and poetry and similarly, different studies have been concerned with evaluating the usability of machine translation systems of literature in different languages. In their experimental study of machine translation systems of literature in Spanish and Catalan, Toral and Way [22] indicated that machine translation systems can be advantageous in this regard. Thenceforth, they applied it to translate one of his novels between two closely related languages (Spanish to Catalan). They discovered that for $20 \%$ of the sentences, the translations produced by the MT system and the professional translators were identical. Moreover, it has been found that over $60 \%$ of the sentences produced by using machine translation systems were of similar quality to that of the professional translator. Similarly, Jones and Irvine [24] evaluated different machine translation systems in translating French prose and poetry. They reported that machine translation systems can be usefully used in literary translation. They concluded that machine translation has respectable potentials for users in relation to literary translation. On the other hand, Besacier [25] indicates that machine translation systems are not effective when it comes to literary texts. To evaluate the effectiveness of machine translation systems of literature, he conducted a study examining the machine translation of a short story to be translated from English into French. He reported that different lexical, syntactic, and structural features were made which had negative effects on the quality of the translation. He also points out that certain cultural references were not of consideration. Henceforth, he comes to the conclusion that machine translation systems of literature are not reliable. This comes in agreement with Kelly and Zetzsche [26] arguments that there are different problems and challenges associated with the machine translation of literature which make it difficult for users to rely on machine translation systems.

In spite of the extensive literature on the assessment of machine translation systems in the Western languages and other languages, The literature indicates that very little has been done, presently, in relation to evaluating machine translation systems of literature in Arabic and the implications of these applications to the EFL contexts. This study addresses this gap in literature by evaluating the machine translation systems of literary texts from English into Arabic. 


\section{$3 \quad$ Methodology}

In order to evaluate the machine translation of literary texts from English into Arabic, two applications were used, namely, Google Translate and QTranslate. The rationale is that the two systems are among the most widely used online translation services. Google Translate is a free online application that can be used to translate texts, words and sentences. It is characterised through its ability to translate for more than seventy languages and is now one of the most widely used machine translation applications all over the world. One problem with this application, however, is that it is limited by the number of characters for every translation activity. QTranslate is also online free translator. However, it has the advantage of searching in online dictionaries and in offline XDXF dictionaries.

The study is based on two prose fiction texts. The first is J. K. Rowling's novel Harry Potter and the Philosopher's Stone (1997). The second is Edgar Allan Poe's short story The Black Cat (1843). The two short stories were translated using Google Translate and QTranalate and the machine translations were compared to human made translations. The former is translated by Sahar Jabr Mahmoud (Mahmoud, 2002) and the latter is translated by Khalda Said (Said, 1986).

Macrotextual methods were used to evaluate the automatic translations of Google and QTranslate. These evaluation methods are concerned with evaluating the function, purpose, and effect of the automatic translations. For convenience purposes, human evaluation is used. The rationale is that manual evaluation is more reliable.

\section{$4 \quad$ Analysis and Discussions}

The findings indicate that both Google and QTranslate commit different errors. These can be summarized as follows: (1) context and usage are usually ignored in machine translations, (2) decoding problems: In decoding, the main reasons are: (a) Languages may code information only partially, and (b) Languages may code information at an arbitrarily long distance, (3) syntactic ambiguity, (4) semantic ambiguity, (5) lexical gaps, (6) discontinuous expressions, (7) pragmatic problems, (8) unknown words, and (9) non-translated sentences.

In the following table, we will present the total errors when using Google and QTranslate applications as well as the non-translated sentences concerning the translation of the two stories; The Black Cat story by Rowling's novel Harry Potter and the Philosopher's Stone.

\begin{tabular}{|l|c|c|c|c|}
\hline & Lexical errors & Structural errors & $\begin{array}{c}\text { Semantic and pragmatic } \\
\text { errors }\end{array}$ & Non-translated sentences \\
\hline Google & 108 & 72 & 54 & 15 \\
& $43.37 \%$ & $28.91 \%$ & $21.68 \%$ & $6.20 \%$ \\
\hline QTranslate & 228 & 156 & 102 & 30 \\
& $44.18 \%$ & $30.23 \%$ & $19.76 \%$ & $15.81 \%$ \\
\hline
\end{tabular}


As shown in the above table, Google application lexical errors (43.37\%) while QTranslate $(44.18 \%)$. Also, the table explains that Google structural errors $(28.91 \%)$ while QTranslate $(30.23 \%)$. Moreover, the graph shows that Google semantic and pragmatic errors $(21.68 \%)$ while QTranslate $(19.76 \%)$. Finally, concerning non-translated sentences, the graph explains that Google non-translated sentences $(6.20 \%)$ while QTranslate (5.81\%).

These errors have consequently negative impacts on the translation quality and reliability. One major problem with these translations is the lack of cohesion and coherence. This agrees with the Voigt and Jurafsky's [27] argument that machine translation systems cannot address issues of cohesion and coherence. It was also found that the automatic translations have a lot of reference chains, thus, the incorporation of discourse features within sentence level is very important to apply machine translation to the literary text. It was also clear that machine translation systems provide translations without considering cultural and contextual factors, which are essential in literary translation. It was also discovered that linguistic peculiarities have a negative impact on the effectiveness of automatic translations. In other words, translation systems compare numerous collections of texts and draw translation rules from them. The flexibility of word order within the Arabic language makes it difficult for translation systems to make correct choices which has distinctive, negative impacts on the effectiveness of these translations.

In spite of these errors, machine translation systems should not be underestimated or rejected. This also does not mean or insinuate that translation technologies should be disregarded in translation classes. The fact is that not all professional translators are good literary translators. I mean literary translation is challenging for many human and professional translators. So far, literary translation is not a job for which MT systems have been designed.

The implication here is that translation students should be aware of these error types of automatic translations. They should be taught that despite the usefulness of these tools in some cases, their limitations should not be overlooked. Translation students should be taught that translation technologies are certainly useful to some extent, however; post-editing of the automatic translations is important in order to address the translation errors usually made by the translation systems.

\section{Conclusion}

In conclusion, it is worth significantly highlighting that there is clearly a benefit to both methodologies for translation, despite their shortcomings. Firstly, the use of machine translators provides a basic, if not, high, level of translation and furthermore, postulates quite a formidable tool for use of translation in both prose and literature. However, as we have seen through the use of in-depth analysis of the two aforementioned novels, there can be serious flaws to machine translation. Conversely, the use of human intervention, specifically, in regard to translation, can provide, evidently, the most accurate form of translation, with respect to correct translation of idioms, grammar and colloquial understandings. However, as mentioned, this is highly dependent upon 
the individual translator and more significantly, the level of their abilities. Thus, it makes it highly understandable, in the aim of accuracy and completeness, that the two methodologies can be used hand in hand for the ultimate perfection. Nonetheless, the individual use of both methods can be highly successful depending on the type of novel or writing that is to be translated, for example, the more colloquial the documents, the less likely a machine translator is to be able to translate accurately and with the utmost reliability; as colloquialism and idioms have a tendency to not to follow the strict grammatical rules or the general structure, as in the standard language. Thus, in this instance a human translation may be favoured. Accordingly, it would be apparent that the teachings of both machine translation and human translation are a necessity for both translation and the improvement, betterment and development of machine translation with the aim of precision in reliability, coherence and cohesion.

\section{Acknowledgment}

We take this opportunity to thank Prince Sattam Bin Abdulaziz University in Saudi Arabia alongside its Deanship of Scientific Research, for all technical support it has unstintingly provided towards the fulfillment of the current research project.

\section{$7 \quad$ References}

[1] J. Moorkens, S. Castilho, F. Gaspari, and S. Doherty, Translation Quality Assessment: From Principles to Practice. Springer International Publishing, 2018. https://doi.org/10. 1007/978-3-319-91241-7

[2] A. Trujillo, Translation Engines: Techniques for Machine Translation. Springer London, 2012.

[3] Y. Wilks, Machine Translation: Its Scope and Limits. Springer US, 2008.

[4] S. Yao, "Application of Computer-aided Translation in English Teaching," International Journal of Emerging Technologies in Learning (iJET) vol. 12, no. 8, pp. 105-117, 2017.

[5] J. Jiang, Y. Qin, and Y. Sun, "Constructing Automated Scoring Model for Human Translation with Multidisciplinary Technologies," International Journal of Emerging Technologies in Learning (iJET), vol. 11, no. 2, pp. 57-62, 2016. https://doi.org/10.3991/ijet.v11i02. $\underline{5137}$

[6] L. Bowker, Computer-aided Translation Technology: A Practical Introduction. University of Ottawa Press, 2002.

[7] K. Malmkjær, Translation in Undergraduate Degree Programmes. J. Benjamins Publishing Company, 2004.

[8] L. Venuti, Teaching Translation: Programs, courses, pedagogies. London; New York: Routledge, 2016.

[9] M. Tymoczko, Enlarging Translation, Empowering Translators. Taylor \& Francis, 2014.

[10] Z. Erwen and Z. Wenming, "Application of Computer-Aided Translation Technology in Translation Teaching," International Journal of Emerging Technologies in Learning (iJET), vol. 8, no. 5, pp. 15-20, 2013. https://doi.org/10.3991/ijet.v8i5.2926

[11] D. Robinson, Becoming a Translator: An Introduction to the Theory and Practice of Translation. Taylor \& Francis, 2004.

[12] S. Chan, The Human Factor in Machine Translation. Taylor \& Francis, 2018. 
[13] M. Sofer, Translator Self-Training Program, Arabic. Schreiber Pub., 2002.

[14] W. Shang, "Construction and Application of WeChat Learning Platform in "Folk Literature" Teaching," International Journal of Emerging Technologies in Learning (iJET), vol. 11, no. 5, pp. 10-15, 2016. https://doi.org/10.3991/ijet.v11i05.5688

[15] N. Eteokleous and D. Ktoridou, "Investigating Mobile Devices Integration in Higher Education in Cyprus: Faculty Perspectives," International Journal of Inter-active Mobile Technologies, vol. 3, no. 1, pp. 38-48, 2009. https://doi.org/10.3991/ijim.v3i1.762

[16] C. Hauenschild and S. Heizmann, Machine Translation and Translation Theory. De Gruyter, 2011.

[17] C. K. Quah, "Machine Translation Systems," in Translation and Technology, C. K. Quah, Ed. (Palgrave Textbooks in Translating and Interpreting, London: Pal-grave Macmillan, 2006.

[18] L. C. Tong, "Translation: Machine-aided " in The Encyclopedia of Language and Linguistics, R. E. Asher, Ed. Oxford: Pergamon Press LTD, 1994, pp. 4730-4737.

[19] H. L. Somers, Computers and Translation: A Translator's Guide. John Benjamins Publishing Company, 2003.

[20] L. Besacier and L. Schwartz, "Automated translation of a literary work: a pi-lot study," in Proceedings of NAACL-HLT Fourth Workshop on Computational Linguistics for Literature, Denver, Colorado, 2015 pp. 114-122. https://doi.org/10.3115/v1/w15-0713

[21] A. Toral and A. Way, "Translating Literary Text between Related Languages using SMT," in Proceedings of NAACL-HLT Fourth Workshop on Computational Linguistics for Literature, Denver, Colorado, 2015, pp. 123-132. https://doi.org/10.3115/v1/w15-0714

[22] A. Toral and A. Way, "Machine-assisted translation of literary text: A case study," Translation Spaces, vol. 4, pp. 240-267, 01/01 2016. https://doi.org/10.1075/ts.4.2.04tor

[23] K. Taivalkoski-Shilov, "Ethical issues regarding machine(-assisted) translation of literary texts," Perspectives, vol. 27, no. 5, pp. 689-703, 2019/09/03 2019. https://doi.org/10.1080/ 0907676x.2018.1520907

[24] R. Jones and A. Irvine, "The (Un)faithful Machine Translator," in Proceedings of the 7th Workshop on Language Technology for Cultural Heritage, Social Sci-ences, and Humanities, 2013, pp. 96-101.

[25] L. Besacier, "Traduction automatis'ee d'uneoeuvre litt'eraire: une'etude pilote," in Traitement Automatique du Langage Naturel (TALN)Marseille, France, 2014.

[26] N. Kelly and J. Zetzsche, Found in Translation: How Language Shapes Our Lives and Transforms the World. Tarcher Perigee, 2012.

[27] R. Voigt and D. Jurafsky, "Towards a Literary Machine Translation: The Role of Referential Cohesion," Proceedings of the NAACL-HLT 2012 Workshop on Computational Linguistics for Literature, pp. 18-25, 2012.

\section{Authors}

Abdulfattah Omar is an Associate Professor of linguistics in Prince Sattam Bin Abdulaziz University. He finished his $\mathrm{PhD}$ in linguistics at Newcastle University in 2010. His research interests include computational linguistics, digital humanities, and literary computing. He is also a faculty member in the Department of English, Faculty of Arts, Port Said University since 2011.

Yasser A. Gomaa is an Associate Professor of linguistics in the University of Bahrain. His research interests focus on sociolinguistics, discourse studies, and computer- 
assisted language learning (CALL). He is also an Associate Professor of English language and linguistics in the Department of English, Faculty of Arts, Assiut University. Email: gomaa@aun.edu.eg

Article submitted 2020-01-20. Resubmitted 2020-02-20. Final acceptance 2020-02-21. Final version published as submitted by the authors. 